\title{
FUNDAMENTOS TEÓRICOS DEL PENSAMIENTO COMPLEJO DE EDGAR MORIN
}

\author{
Paulette Barberousse $e^{1}$ \\ Profesora e investigadora del CIDE-Universidad Nacional \\ Heredia, Costa Rica
}

\begin{abstract}
"Nunca pude, a lo largo de toda mi vida, resignarme al saber parcelado, nunca pude aislar a un objeto de estudio de su contexto, de sus antecedentes, de su devenir. He aspirado siempre a un pensamiento multidimensional. Nunca he podido eliminar la contradicción interior. Siempre he sentido que las verdades profundas, antagonistas las unas de las otras, eran para mí complementarias, sin dejar de ser antagonistas. Nunca he querido reducir a la fuerza la incertidumbre y la ambigüedad".
\end{abstract}

Edgar Morin ${ }^{2}$

Recibido: 2 de noviembre, 2007 • Aprobado 1어이 de diciembre, 2007

Resumen: Ante lo que consideró la crisis del paradigma occidental de simplificación y disyunción, basado en la reducción y separación de los saberes, Edgar Morin planteó la emergencia de un nuevo paradigma de la complejidad, el cual intentaría articular y contextualizar las culturas científicas, las culturas de las humanidades y las culturas artísticas. Para llevar adelante tal propósito, se basó en la integración de ideas, conceptos y nociones provenientes de diversas fuentes teóricas.

El abordaje de la complejidad ha requerido de la resignificación dialéctica y creativa del legado de dichas teorías en una nueva síntesis que, al mismo tiempo que las integra, las eleva a un nivel cualitativamente distinto y original.

Palabras clave: Pensamiento complejo, teoría de sistemas, teoría cibernética, teoría de la información y comunicación, legado piagetiano, paradigma kuhniano.

Abstract: In face of what he considered as the crisis of the Western paradigm of simplification and disjunction, based on the reduction and separation of knowledge, Edgar Morin posited the emergence of a new paradigm of complexity that would attempt to articulate and contextualize scientific, humanistic, and artistic cultures. To accomplish such purpose, Morin argued for an integration of ideas, concepts and notions drawing on different theoretical sources.

Approaching complexity has required a dialectic and creative resignification of the legacy of such theories through a new synthesis that both integrates and surpasses them qualitatively.

Key words: Complex thinking, systems theory, cybernetics theory, information and communication theory, Piagetian legacy, Kuhnian paradigm. 


\section{Introducción}

La irrupción del paradigma de la complejidad y la propuesta del pensamiento complejo de Edgar Morin, en el paisaje científico contemporáneo, ha provocado, sin duda, un replanteamiento de los fundamentos mismos de la racionalidad occidental. Su repercusión en el campo pedagógico se ha evidenciado en la medida que pone entre paréntesis los conceptos conocimiento y aprendizaje al cuestionar sus fundamentos epistémicos. Ha planteado, además, la necesidad de reformar el pensamiento $y$, al mismo tiempo, reformar las instituciones educativas, con el fin de promover una educación acorde con las necesidades de la sociedad contemporánea.

La propuesta del pensamiento complejo ha cuestionado no sólo el concepto mismo de ciencia, sino que ha proclamado que lo que ha variado es la naturaleza misma de lo que entendemos por conocimiento. En consecuencia, se verían también afectados los procesos de construcción de saberes y aprendizajes que ocurren en el marco de las mediaciones pedagógicas.

No solamente se han modificado los soportes físicos del conocimiento (antes consultábamos en los gruesos y voluminosos tomos de la Enciclopedia Británica, actualmente están disponibles en Internet), sino que hoy entendemos el conocimiento como abierto, inconcluso, siempre relativo y en permanente reconstrucción.

La intencionalidad de este trabajo se basa en la descripción reflexiva de los principales fundamentos teóricos de la propuesta del pensamiento complejo de Edgar Morin y tiene como principal propósito contribuir al debate sobre las ideas-fuerza que conforman la propuesta moriniana, enfatizando en aquellos aspectos que hemos creído han constituido un salto cualitativo con respecto a sus predecesores.

Se pretende contribuir en el debate acerca de los principales supuestos teóricos de la propuesta del pensamiento complejo de Edgar Morin, en la medida en que pueden ayudarnos a replantear las bases epistémicas que han sustentado las posturas pedagógicas que fundamentan el quehacer académico y educativo. 


\section{¿Qué hemos entendido por sistemas complejos?}

En este tercer milenio, y en el seno de una sociedad globalizada, neoliberal y posmoderna, la complejidad de los nuevos modelos y teorías científicas nos obligó a considerar a la información como un insumo de gran valor. La revolución científico-tecnológica ha suscitado cambios profundos en la sociedad contemporánea, fundamentalmente cambios en las mentalidades, perspectivas y valores humanos.

En este contexto el paradigma mecánico vigente asumió la dicotomía "dato-información", entendiendo al primero como objetivo y al segundo como subjetivo. Por el contrario, un abordaje sistémico desde el paradigma de la complejidad eliminó esta falsa dicotomía y acentuó los elementos comunes de, por lo menos, cuatro aspectos: la información subjetiva, la información objetiva, el proceso empírico de sensaciones-percepciones y las acciones sobre la información, considerándolas en un complejo dialéctico. La información se muestra como comprensiva, ya que integra todos los elementos anteriormente mencionados, como estructurada por los mismos cuatro elementos y como distribuida desde el punto de vista de la verdad pragmática.

La construcción de realidades e instrumentos virtuales constituyó un proceso de “desmaterialización" de los procesos mentales. El aumento de la capacidad de procesamiento de información, asociada con la creciente especialización y complejidad de las estructuras bioquímicas y físicas del soporte de la información, constituyó un salto cualitativo profundo en la cosmovisión del mundo en el cual vivimos.

El ser humano ha logrado interactuar de una manera eficiente, y cada vez más inteligente, con su entorno, y aumentó su capacidad de reflejar, creativamente, la realidad por medio de cálculos y modelos formales. Los nuevos procesamientos de la información provocaron la aparición de lenguajes y metalenguajes que estructuraron la mente de quien los utilizó y cambió, drásticamente, nuestro modo y estilo de vida.

En este proceso de construcción humana, el conocimiento adquirió una nueva dimensión $y$, al transformarse en poder, nos definió un nuevo cuadro ontológico que se basó en la tríada: información-energía-materia. 
El concepto de sistema complejo se fundamentó en esta visión triádica, la cual nos permitió construir un abordaje estructural-fenoménico y heurístico de los procesos y fenómenos de la realidad.

El paradigma de la complejidad nos aseguró un marco conceptual que permitió establecer interrelaciones e intercomunicaciones reales entre las diversas disciplinas, y provocó un fecundo diálogo entre especialistas, metodologías y lenguajes específicos.

Cobijados bajo el manto del paradigma de la complejidad los sistemas complejos, según Morin (1994), se basaron, primordialmente, en un abordaje no-lineal de la realidad y se afirmaron, entre otros, en los siguientes enunciados:

- un sistema complejo no puede ser analizado, en principio, en forma fragmentaria, es decir, por partes; se halla constituido por un sistema de elementos que tiene un/múltiples sentido/s en la intimidad del sistema considerados en un horizonte temporal limitado y puede sufrir transformaciones y cambios bruscos;

- $\quad$ se diferenció de un sistema complicado, dado el hecho de que la dificultad de predicción no se encontró en la incapacidad del observador de tener en cuenta todas las variables que influyeron su dinámica, sino en la "sensibilidad" del sistema a las condiciones iniciales (debemos tener en cuenta que las condiciones iniciales diferentes condujeron a evoluciones extremadamente diversas) a las cuales se agregaron los efectos de los procesos de autoorganización (procesos que se hallaron condicionados por las interacciones de los subsistemas componentes, y que tuvieron como efecto la aparición espontánea y no predecible de unas determinadas relaciones de orden);

- un sistema complejo incorporó una evolución que no resultó del análisis de sus respuestas a un estímulo dado (bajo la forma de un análisis dinámico), sino que su dinámica y su evolución necesitaron de abordajes específicos y distintos. 
Morin nos explica en su libro El método I (1993), que:

\begin{abstract}
Un sistema es una interrelación de elementos que constituyen una entidad global o unidad global. Tal definición comporta dos caracteres principales: el primero es la interrelación de los elementos y el segundo es la unidad global constituida por estos elementos en interacción (...) se puede concebir el sistema como unidad global organizada de interrelaciones entre elementos, acciones o individuos (pp. 123-124).
\end{abstract}

Morin (1994) subrayó el hecho de que un sistema complejo, entendido como unidad global, puede manifestar propiedades que no pueden ser explicadas a partir de sus componentes. El todo manifestó propiedades emergentes, colectivas, propiedades que no tenían ningún significado para la dinámica de sus partes componentes.

La complejidad apareció, justamente, como resultado de la organización del todo bajo la presión de las infinitas combinaciones de interacciones simultáneas, y que abundaron en interrelaciones no-lineales. La complejidad nació de la interacción de las partes que lo componen, es decir, la complejidad se manifestó en el sistema mismo.

Según Pozo (2001) las teorías científicas han favorecido nuestra comprensión de la realidad y han operado con nociones "simplificadas" que nos han permitido estructurar nuestros conocimientos en función de un reducido número de variables que nos han facilitado la construcción de modelos explicativos de sistemas lineales que nos han "aproximado" a la realidad, una realidad mutilada en su complejidad.

Asume el autor que el abordaje desde la perspectiva de la complejidad ha considerado la noción de complejo como cualitativamente diferente. La realidad se ha comprendido como un conjunto de sistemas jerarquizados constituidos por subsistemas que interactúan en forma permanente, asimilan informaciones, aprenden y cambian sus comportamientos. Además, se adaptan a las modificaciones de su entorno mediante procesos que podríamos calificar de coevolutivos y que han implicado, en el caso del ser humano, necesariamente, los procesos de construcción de conocimientos y de aprendizajes. 


\title{
¿Cuáles han sido los fundamentos teóricos del pensamiento complejo?
}

\author{
Dejemos que sea el propio Morin (1994), el que nos confiese, cuáles han sido los
} principales fundamentos teóricos de su propuesta epistémica:

\begin{abstract}
Desde mis primeros libros he afrontado a la complejidad, que se transformó en el denominador común de tantos trabajos diversos que a muchos le parecieron dispersos. Pero la palabra complejidad no venía a mi mente, hizo falta que lo hiciera, a fines de los años 60 , vehiculizada por la Teoría de la información, la Cibernética, la Teoría de sistemas, el concepto de auto-organización, para que emergiera bajo mi pluma o, mejor dicho, en mi máquina de escribir. Se liberó, entonces, de su sentido banal (complicación, confusión) para reunir en sí orden, desorden y organización y, en el seno de la organización, lo uno y lo diverso; esas nociones han trabajado las unas con las otras, de manera a la vez complementaria y antagonista; se han puesto en interacción y en constelación. El concepto de complejidad se ha formado, agrandado, extendido sus ramificaciones, pasado de la periferia al centro de mi meta, devino un macro-concepto, lugar crucial de interrogantes, ligando en sí mismo, de allí en más, al nudo gordiano del problema de las relaciones entre lo empírico, lo lógico y lo racional (pp. 23-24).
\end{abstract}

De las fuentes de donde abrevó el pensamiento complejo de Morin podemos citar, a saber:

\section{Teoría de sistemas}

Hemos creído importante rescatar, en palabras del propio Morin (1994), su apreciación sobre la Teoría de sistemas, nos ha dicho lo siguiente:

\footnotetext{
La Teoría de sistemas y la Cibernética se recortan en una zona incierta común. En principio, el campo de la Teoría de Sistemas es mucho más amplio, casi universal, porque en un sentido, toda realidad conocida, desde el átomo hasta la galaxia, pasando por la molécula, la célula, el organismo y la sociedad, puede ser concebido como sistema, es decir, como asociación combinatoria de elementos diferentes. De hecho, la Teoría de Sistemas que empezó con Bertalanffy como una reflexión sobre la Biología, se expandió frondosamente, a partir de los años 1950, en las más variadas direcciones (pp. 41-42).
} 
$\mathrm{Y}$, con respecto a los aportes de la sistémica, reflexiona:

La virtud sistémica es:
a) haber puesto en el centro de la teoría, con la noción de sistema, no una unidad discreta, sino una unidad compleja, un "todo" que no se reduce a la "suma" de sus partes constitutivas.
b) haber concebido la noción de sistema, no como una noción "real", ni como una noción puramente formal, sino como una noción ambigua o fantasma.
c) situarse en un nivel transdisciplinario que permite concebir, al mismo tiempo, tanto la unidad como la diferenciación de las ciencias, no solamente según la naturaleza material de su objeto, sino también según los tipos y complejidades de los fenómenos de asociación/organización. En este último sentido, el campo de la Teoría de Sistemas es, no solamente más amplio que el de la Cibernética, sino de una amplitud que se extiende a todo lo cognoscible (p. 42).

Por tanto, a comienzos del siglo $\mathrm{XX}$, se instauró la perspectiva sistémica que continúa hasta hoy considerada como un instrumento con una fuerte posibilidad heurística. Al biólogo Ludwing Von Bertalanffy se le ha considerado el padre de la Teoría General de Sistemas, y nos mostró una nueva forma de abordaje de la realidad que prevalece hasta nuestros días.

De acuerdo con Agazzi (1996), citado por Moreno (2002), esta teoría asumió que para poder pensar lo sistémico tenemos que ubicarnos en el contexto del pensamiento relacional. Bertalanffy (1974) buscó explicitar los aspectos de unidad que caracterizan un cierto sistema organizado de elementos y lo denominó conjunto de elementos, es decir, un sistema. Desde una perspectiva holística, asumió que el sistema goza de propiedades emergentes, se halla constituido por subsistemas e interacciona con suprasistemas.

Según Moreno (2002) la idea de sistema permitió pensar esas totalidades como relacionadas -un sistema se constituye como tal en relación con subsistemas y suprasistemas y los cambios afectan a todos-; como irreductibles -a pesar de estar relacionados no pierden su unidad, identidad y autonomía ya que poseen una organización interna específica-; como dinámicas -ya que si bien los sistemas tienden entrópicamente a la desorganización, es decir, hacia su muerte y desaparición, logran mantener su equilibrio interno a través de procesos 
neguentrópicos de reorganización y de adaptación a las perturbaciones-; como adaptables -se adaptan a las perturbaciones tanto de sub como de suprasistemas y mantienen su organización interna-; como cambiantes dado que las propiedades emergentes provocan cambios permanentes y nunca un sistema queda absolutamente definido o adaptado.

Sin duda, Bertalanffy (1974) fue consciente, del carácter radical que involucraba su perspectiva holística, ya que su teoría general es aplicable a cualquier campo y constituía un cambio paradigmático en la ciencia. Sus ideas fundamentaron la visión de los organismos vivos como sistemas abiertos y posibilitó la asunción del concepto de autoorganización y que ha significado que hablemos hoy de una teoría de sistemas autoorganizadores.

En la sistémica clásica se tendió siempre hacia el equilibrio del sistema, es decir, los desórdenes de origen entrópico o de origen externo logran ser compensados, de manera neguentrópica, mediante una retroalimentación ${ }^{3}$ negativa para que el sistema no pierda su organización. Los procesos de adaptación y cambio siempre se han dado hacia el equilibrio y motivados no por el mismo sistema, sino sólo como respuesta a lo que lo amenaza (como lo plantea la propuesta piagetiana).

Sin duda, esta concepción -tal como nos lo detalla Moreno (2002)- significó un salto cualitativo con respecto a la explicación mecanicista pura, pero si este mismo proceso quiere ser aplicado a los seres vivos no es suficiente explicar cómo los seres vivos se adaptan y logran su equilibrio frente a una amenaza, sino cómo éstos cambian hacia nuevas formas de organización o cómo se transforman desde sí mismos (y no como respuesta reactiva a algo) en nuevos sistemas. En última instancia, estamos hablando de explicar el cambio mismo, es decir, cómo abandona su organización interna que los identifica como sistemas y asumen otra organización interna construida por ellos mismos que los reconvierte, nuevamente, en otro sistema que guarda algo del anterior, pero anuncia ya su propia reorganización futura.

Es en este sentido que en su obra El Método I, Edgar Morin (1993) con respecto a la teoría de sistemas, nos aclara que:

\footnotetext{
${ }^{3}$ Se mantiene esta grafía por ser la utilizada originalmente por Morin
} 
la teoría del sistema se anima allí donde hay un juego activo de interacciones, retroacciones, emergencias, constreñimientos; allí los antagonismos entre las partes, entre las partes y el todo, entre lo emergente y lo sumergido, entre lo estructural y lo fenoménico, se ponen en movimiento (p. 179).

Además, en lo referente a la relación elementos/organización propios de todo sistema, Morin (1993) nos planteó que:

Los elementos deben ser definidos, pues, a la vez y por sus caracteres originales, en y con las interrelaciones de las que participan, en y con la perspectiva de la organización en la que están dispuestos, en y con la perspectiva del todo en el que se integran. Inversamente, la organización debe definirse con relación a los elementos, a las interrelaciones, al todo, y así sin interrupción. El circuito es polirrelacional (p. 151).

Sin embargo, este autor ha insistido, permanentemente, en todo su discurso que la inclusión de la visión sistémica no ha implicado que su propuesta haya caído en un holismo indeterminado y general que significaría, de hecho, la contrapartida opuesta al reduccionismo propuesto por el paradigma mecanicista, sólo que de signo contrario (el todo existe, pero también existen las partes).

\section{Teoría cibernética}

Comencemos por explicar que fue el matemático y físico Norbert Wiener (1894-1964) quien elaboró el concepto de cibernética en su obra Cibernética y sociedad. La palabra cibernética proviene del término griego kybernetes que, según el Diccionario de la Real Academia Española significa "arte de gobernar una nave". Agrega que la cibernética es el "estudio de las analogías entre los sistemas de comunicación y control de los seres vivos y de los de las máquinas, en particular, el de las aplicaciones de los mecanismos de regulación biológica a la tecnología".

Ashby (1997), citado por Moreno (2002), se refirió a ello del siguiente modo: 
(...) aunque han aparecido muchos libros titulados Teoría de las máquinas (sic), éstas, generalmente, aluden a objetos mecánicos, levas y palancas, por ejemplo. También la cibernética es una "teoría de las máquinas", pero no estudia objetos, sino modos de comportamiento. No pregunta ¿qué es esto?, sino, ¿qué hace? (...) Es, por lo tanto, esencialmente funcional y conductista (...) trata todas las formas de conducta en la medida que son determinables, regulares o reproducibles (pp. 11-12).

Otros dos conceptos básicos de la cibernética los han constituido el de causalidad circular, fundamento en el nivel lógico que torna comprensible el fenómeno físico de la retroalimentación (feedback) concebida como constante fluir de información del ser vivo con su ambiente.

Para la propuesta moriniana, los conceptos de causalidad circular y retroalimentación, que provienen del campo cibernético, resultan imprescindibles para la construcción del principio de auto-eco-organización. Este principio ha fundamentado que los fenómenos no deben ser concebidos aislados de sus entornos, ni como producto de determinaciones externas, sino que deben tener en cuenta una dialógica compleja de doble implicación entre la lógica interna del sistema y la lógica externa de la situación o entorno. Todo fenómeno debe ser considerado en su ecosistema: el pensamiento complejo ha exigido considerar al pensamiento como ecologizado, en su relación coorganizadora con su ambiente.

\section{Teorías de la información y la comunicación}

Surgió a partir del trabajo de Shannon y Weaver (1981) conocido como Teoría matemática de la información. Según Moreno (2002) su objeto de estudio se centraba en el análisis de la eficacia de la información y buscaba establecer medidas cuantitativas sobre la capacidad de los sistemas de transmitir, almacenar y procesar información. Intentaron -según el autor citado- descubrir las leyes matemáticas que gobiernan la información y establecer la medida cuantitativa mínima que reduce la incertidumbre de un mensaje.

De acuerdo con Morin (1994) no fue sino con la incorporación del concepto de retroalimentación positiva de la cibernética a los procesos de comunicación, con lo cual se pasó 
de una teoría de la información lineal a una circular. Su principal representante Gregory Bateson en su libro Comunicación, la matriz social de la psiquiatría proclamó que todas las experiencias, y expresiones humanas se hallan mediadas por la comunicación.

Morin (1994) asumió los supuestos teóricos de la teoría comunicacional en la medida que consideró el lenguaje y la comunicación humana (en el sentido más extenso del término) como el primer modo de representación simbólica de la realidad. Junto con el desarrollo de la praxis humana sociohistórica (por medio de las actividades productivas) se desarrollan y se conforman mutuamente, las estrategias y las modalidades del pensamiento y del lenguaje. En esta tríada interrelacionada de trabajo-lenguaje-pensamiento -que nos viene dada por la concepción marxista, pasando por Luria y Vigotsky- se fundamentó Morin para explicar la conformación del sujeto cognoscente y epistémico.

\section{Legado piagetiano}

Morin (1994) reconoce que el legado piagetiano ocupó un lugar central en la articulación de su propuesta. Nos aclara que:

\footnotetext{
(Jorge Correia Jesuino) ha señalado mi insuficiente atención en relación con Piaget. Estoy de acuerdo. Es por razones, a la vez aleatorias y contingentes, que le he dado tan poco lugar explícito a Piaget. Ante todo, los autores que han sido citados en abundancia en mi trabajo son aquellos que yo he descubierto después de 1968, y sobre los cuales yo tomaba notas en relación con El Método. Conocía a Piaget desde antes (...), así es que Piaget parece subestimado en mis libros siendo que es un autor crucial. Se ubica en el cruce de caminos entre las ciencias humanas, la biología, la psicología y la epistemología (pp. 157-158).
}

Al analizar las trayectorias de vida de ambos hemos podido encontrar múltiples puntos de encuentro: los dos son europeos, uno suizo, el otro francés; ambos son, relativamente, contemporáneos, sólo los separa una generación (Piaget es el de edad más avanzada), a pesar de que lo más fecundo de sus propuestas epistémicas se construyen en un relativo paralelismo en cuanto al espíritu y preocupaciones de época; ambos son epistemólogos y sus temas fundamentales de interés han girado en torno a los procesos de construcción de conocimientos 
y saberes; por último, sus planteamientos constructivistas han poseído el potencial epistémico para traducirse en planteamientos pedagógicos (tal como ha ocurrido en el caso de Piaget con la pedagogía operatoria y, en general, con las propuestas del constructivismo pedagógico).

Vamos a rescatar tres conceptos que hemos creído medulares y que son compartidos, pero que, a la vez, marcan sus particulares puntos de vista.

\section{- Noción del "circuito de las ciencias" de Piaget}

Esta noción ha establecido la interdependencia de facto de las diversas ciencias y planteó la necesidad de integración de las ciencias naturales y humanas. De hecho, Piaget asumió que el ser humano es un ser biológico, físico y también cultural. La crítica de Morin (2002), con respecto a este concepto, se basó en el hecho de que a su mirada se le escapa "la interarticulación entre ciencias que tienen, no sólo un lenguaje propio, sino conceptos fundamentales que no pueden pasar de un lenguaje a otro" (p. 124).

Morin (2002) plantea que el circuito de las ciencias de Piaget debió integrarse en una concepción que él denomina circuito epistemológico, que no sólo ha promovido la fusión holística de las ciencias naturales, humanas y artísticas, sino que asumió que el camino de esa integración pasa por procesos de búsqueda inter y transdisciplinaria.

\section{- $\quad$ La noción de sujeto epistémico de Piaget}

Partamos de la base de que quien construye la ciencia es el ser humano e involucra en esta acción su dimensión subjetiva que transmite, además, su postura epistémica. Ambos enfoques se integran en una unidad en diversidad y constituyen facetas indisolubles a la hora de la construcción de saberes y conocimientos.

Jean Piaget intentó explicar la génesis y la transformación del conocimiento científico, en el marco de una psicología cognitiva, específicamente desde la epistemología genética. 
Propuso que la construcción de las estructuras cognitivas dependían de los esquemas de acción y de las operaciones mentales. Determinó que las funciones cognitivas básicas se apoyan en procesos de organización y adaptación de los organismos vivos mediante procesos de equilibración que implicaron, a su vez, mecanismos de asimilación y acomodación de los llamados contenidos de la cognición que pautaron las, ya famosas, etapas de desarrollo cognitivo.

Si bien Morin (1994) declaró ser partidario del constructivismo piagetiano, agregó que el autor ignora que son "necesarias fuerzas organizacionales complejas innatas para que haya fuertes aptitudes para conocer y aprender" (p. 27). Hace referencia a los planteamientos de Chomsky (con el cual Piaget debatió fuertemente) acerca de la preexistencia de estructuras innatas de la percepción y de la construcción, capaces de ser adquiridas, únicamente, como "fruto de una dialógica con el medio exterior" (Morin, 1994, p. 27).

Jean Piaget manejó el concepto de autorregulación y lo circunscribió sólo al ámbito interno del sujeto, el cual reacciona, adaptativamente, frente al medio y sus perturbaciones, mientras que Morin superó esa visión al incorporar al sujeto en su contexto, es decir, al hablar del principio de autoecoorganización y de ecosistema, en el cual la díada sujeto-contexto fueron considerados en una dialógica mutuamente fundante.

\section{- $\quad$ Origen biológico del conocimiento de Piaget}

Morin (1994) concordó con el origen biológico del conocimiento planteado por Jean Piaget, pero consideró que el autor suizo "permaneció en el nivel de la idea de organización y de regulación, sin acceder a la problemática compleja de la auto-organización" (p. 36).

Morin (1994) planteó que el problema del conocimiento se encuentra en el corazón de la vida y que tal concepción no se opone, por biológica, a la concepción filosófica del conocimiento, ya que el conocimiento se encuentra enraizado, vitalmente, en el sujeto. Piaget intentó explicar las fuentes del conocimiento en la biología, es decir, planteó que los conocimientos tienen, como fuente primigenia, los principios fundamentales de la organización 
viviente. Planteó, así, un isomorfismo estructural entre las organizaciones biológicas y cognitivas, y buscó el origen de los procesos cognitivos en los procesos de asimilación y acomodación del organismo.

Piaget (1967) encontró la autorregulación como punto de partida para la comprensión de los procesos cognitivos y afirma que "Los procesos cognitivos aparecen simultáneamente como los resultados de la auto-regulación organísmica, cuyos mecanismos esenciales reflejan, y como los órganos más diferenciados de esta regulación en el seno de las interacciones con el exterior" (p. 49).

Morin (1994) integró, a esta idea piagetiana de autorregulación, el principio de autoecoorganización. Sin embargo, aclaremos que su idea central giró en torno al hecho de que el sujeto como observador/perceptuador no puede concebirse aislado del objeto estudiado, y se le debe considerar en y por su relación coorganizadora con su ambiente y entorno en el marco de un pensamiento ecologizante.

Para finalizar, supongo, además, que ambos, Piaget y Morin, han sido pensadores irremediablemente, y, tal vez, a pesar de sí mismos: curiosos. Esa curiosidad epistémica fue lo que los impulsó a ser irreverentes y transgresores con lo pactado, con lo socialmente correcto, con lo establecido. Esa curiosidad que los mantuvo y mantiene alertas para autocriticarse y no ser complacientes.

\section{Planteamiento paradigmático de Thomas Kuhn}

El término paradigma ha resultado, desde siempre, polémico y polisémico. A partir de Kuhn (1977) el significado del término ha pendulado entre una interpretación que ha oscilado desde una dimensión teórico-metodológica hasta una interpretación de carácter global.

Según Osorio (2002), Kuhn cuestionó su desarrollo lineal y acumulativo de las ciencias naturales y físicas. Llegó a la conclusión de que el conocimiento científico y la ciencia son un producto social e histórico, y que el investigador no es neutral frente a esa realidad, sino que se 
encuentra condicionado por sus tradiciones disciplinarias y por la institución a la cual pertenece.

De acuerdo a sus postulados, con la ayuda indispensable del lenguaje y culturalmente condicionados (si bien no, absolutamente, determinados), los seres humanos hemos desarrollado relaciones intersubjetivas y hemos construido sentidos y significaciones de nuestra vida y nuestro lugar en el mundo. Nuestra racionalidad se fundamenta en nuestros marcos conceptuales e intereses, en nuestra forma de "leer" la realidad.

Morin (1994) planteó la idea de que la teoría del conocimiento deviene en teoría crítica de la sociedad, ya que el modo como conocemos fundamenta nuestra racionalidad y fundamenta, además, sus principios de inteligibilidad. El paradigma se ha transformado, de esta manera, en una estructura de interpretación de la realidad y de orientación de la acción.

El pensamiento complejo se opuso a la concepción que considera al paradigma únicamente como una herramienta heurística y procuró fundamentar una epistemología en permanente construcción y re-construcción.

Morin (1992) está persuadido de que:

La salida es lógicamente imposible y la lógica no puede sino encerrarnos en un círculo vicioso: hay que cambiar las condiciones socioculturales para cambiar la conciencia, pero hay que cambiar la conciencia para modificar las condiciones culturales. Cada verdadera revolución paradigmática se efectúa en condiciones lógicamente imposibles, pero así ha nacido la vida, así ha nacido el mundo: en condiciones lógicamente imposibles (p. 242).

Y Morin (1992) agrega, más adelante, que los paradigmas:

(...) tienen un sentido generativo y organizacional. En virtud de este sentido generativo y organizacional el paradigma orienta, gobierna y controla los pensamientos de los individuos y el sistema de ideas y teorías. Los paradigmas son, siguiendo a Holton, ideas-fuerza obsesivas que determinan una concepción de mundo. $\mathrm{O}$, siguiendo a Maruyama, son visiones de mundo o mentalidad -"mindscape"-. Por ello, un paradigma controla no sólo las ideas y las teorías, sino también el campo cognoscitivo, intelectual y cultural donde nacen y se reproducen esas ideas. Siguiendo a Foucault, el paradigma es el nudo arqueológico anterior, previo, fundador, modelizador y generador de la organización cognitiva, noológica cultural y social. Dicho de otra manera, el paradigma depende del conjunto de instancias cerebrales, espirituales, computantes, cogitantes, lógicas, lingüísticas, teóricas, mitológicas, culturales y sociales e históricas que de él dependen. Dependen de las actualizaciones que dependen de él (p. 236). 
El paradigma, según Morin (1994), organiza nuestra cosmovisión y, por lo tanto, se debe construir un paradigma que cuestione nuestra racionalidad simplificadora. Es decir, no se limita como en el caso de Kuhn, a restringir al paradigma a su rol de enfoque teórico-metodológico, sino que éste debe transformarse en una herramienta de crítica y cuestionamiento de su propia racionalidad y fundamentos.

Después de realizar esta descripción reflexiva acerca de los supuestos teóricos del pensamiento complejo de Morin podemos asumir las siguientes conclusiones:

- Morin asumió la idea de sistema complejo de la sistémica clásica y le integró la idea de complejidad. Asumió que la complejidad nació de la interacción de las partes que componen al sistema complejo, es decir, la complejidad se manifestó en el sistema mismo. La complejidad apareció justamente como resultado de la organización del "todo" bajo la presión de las infinitas combinaciones de interacciones simultáneas y que abundaron en interrelaciones no-lineares. La complejidad no ha resultado una cualidad adicional, por fuera, de los sistemas complejos, sino, que forma parte constitutiva del mismo.

- Con respecto a la sistémica Morin integró la noción de sistema complejo, en el sentido anteriormente mencionado, y lo concibió en el marco de una teoría de los sistemas autoorganizadores. Propuso que el ser humano se ha constituido como un sistema autoorganizado en la medida que se produce a sí mismo de manera constante y posee una relativa independencia con respecto a su medio o contexto. Presenta una clausura operacional en la medida que se mantiene idéntico organizacionalmente, a sí mismo, a pesar de los cambios y perturbaciones estructurales tanto internos como externos se halla abierto desde el punto de vista informacional con su entorno, con su ecosistema. Esta apertura informacional se ha materializado y objetivado en las múltiples interrelaciones comunicativas que mantiene consigo mismo y con el resto de sus congéneres. El ser humano resulta, por lo tanto, productor y producto de sí mismo. 
- En lo referente a la contribución de la cibernética hemos podido destacar que para la propuesta moriniana los conceptos de causalidad circular y retroalimentación resultan imprescindibles para la construcción del principio de auto-eco-organización. Este principio ha planteado la necesidad de relacionar la lógica interna del sistema con la lógica externa del entorno en una dialógica de doble implicación, de circularidad, de retroalimentación en una relación de conformación mutua, de co-organización entre el sistema y su entorno o ecosistema.

- En relación con el aporte de la teoría comunicacional hemos podido advertir que Morin se sumó a la postura propuesta por Bateson acerca del carácter social de la comunicación humana y la integró en la tríada trabajo-pensamiento-lenguaje, herencia de la tradición marxista, retomada por Luria y Vigotsky. Morin asumió que, en los procesos de hominización y en los de humanización, el sujeto ha desarrollado dialécticamente sus estrategias de pensamiento y raciocinio (destrezas mentales), junto con sus destrezas de trabajo manual con instrumentos y herramientas y sus destrezas comunicacionales en un proceso global e integrado tanto desde el punto de vista ontogenético como filogenético.

- $\quad$ El legado piagetiano se ha revelado como esencial para la arquitectura conceptual de la propuesta moriniana en, al menos, tres aspectos básicos: primero la noción de círculo de las ciencias que fue asumida por Edgar Morin como círculo epistemológico mediante la integración de la idea de búsqueda inter y transdisciplinaria; segundo, la noción de sujeto epistémico auto-regulado para Piaget que se adapta al medio, que se transformó en la idea de sujeto incorporado a su contexto en una dialógica ecologizante de autoconformación sujeto-contexto $y$, tercero, la idea piagetiana de auto-regulación que se integró a la propuesta de un principio auto-eco-organizador en el cual el sujeto observador no puede concebirse aislado del objeto estudiado sino en y por su relación cofundante con su objeto, con su entorno en el marco de un pensamiento contextualizante y ecologizante. 
- $\quad$ Por último, la propuesta paradigmática clásica de Thomas Kuhn ha restringido, según Morin, al paradigma a su papel de "enrutador" teórico-metodológico. Por el contrario su propuesta contempló la posibilidad de que el paradigma se transforme en un instrumento de crítica y transformación de su propia racionalidad y fundamentos toda vez que ha considerado que toda teoría del conocimiento equivale, de hecho, a una teoría de crítica social.

Hemos concluido que la propuesta del pensamiento complejo de Morin ha resignificado de una manera dialéctica y creativa el legado de dichas teorías en una nueva síntesis que, al mismo tiempo que las integra, las eleva a un nivel cualitativamente distinto y original.

Queremos finalizar sumándonos a las ideas expresadas por Marcelo Pakman en la Introducción al libro de Introducción al pensamiento complejo (1994) de Morin, cuando escribe:

En Morin, su producción teórica no es nunca un intento de logro acabado, sino más bien un proceso que, en su devenir mismo, marca un rumbo cognitivo en el que somos invitados a participar. (...) Su obra debe, en consecuencia, ser entendida no sólo en términos de su contenido, sino del proceso productor (pág. 9).

\section{Referencias bibliográficas}

Agazzi, E. (1996). El bien, el mal y la ciencia. Madrid: Tecnos.

Ashby, W. (1997). Introducción a la cibernética. Buenos Aires: Nueva Visión.

Bateson, G. (1984). Comunicación, la matriz social de la psiquiatría. Madrid: Paidós.

Bertalanffy, L. (1974). Robots, hombres y mentes: la psicología en el mundo moderno. Madrid: Guadarrama. 
Gutiérrez, G. (2001). Notas de las Ilamadas crisis de los paradigmas en: Globalización, caos y sujeto en América Latina. El impacto de las estrategias neoliberales y las alternativas. San José, Costa Rica: Ediciones DEI.

Kuhn, T. (1977). La estructura de las revoluciones científicas. México: Fondo de Cultura Económica.

Moreno, J. C. (2002). Tres teorías que dieron origen al pensamiento complejo: sistémica, cibernética e información. En M. A. Velilla (Comp.), Manual de iniciación pedagógica al pensamiento complejo (pp. 25-37). Bogotá: Instituto Colombiano para la Educación Superior-UNESCO.

Morin , E. (1992). El Método IV: Las ideas. (Vol. 4), (Trad. del fr. por Ana Sánchez). Madrid: Cátedra.

Morin, E. (1993). El Método I: La naturaleza de la naturaleza. Madrid: Càtedra.

Morin, E. (1994). Introducción al pensamiento complejo. (Trad. del fr. por Marcelo Pakman). Barcelona: Gedisa.

Morin, E. (2002). La cabeza bien puesta. Repensar la reforma, reformar el pensamiento. Bases para una reforma educativa. Buenos Aires: Nueva Visión.

Osorio, S. N. (2002). Aproximaciones a un nuevo paradigma en el pensamiento científico. En M. A. Velilla (Comp.), Manual de iniciación pedagógica al pensamiento complejo (pp. 38-59). Bogotá: Instituto Colombiano para la Educación Superior-UNESCO.

Piaget, J. (1967). Lógica y conocimiento científico. París: Gallimard.

Pozo, J. I. (2001). Humana mente: el mundo, la conciencia y la carne. Madrid: Morata.

Shannon, C. y Weaver, W. (1981). Teoría matemática de la información. Madrid: Forja.

Wiener, N. (1977). Cibernética o el control y comunicación en animales y máquinas. Madrid: Tusquets. 